\title{
Hybrid Finite-Difference Time-Domain Modeling of Curved Surfaces Using Tetrahedral Edge Elements
}

\author{
Ruey-Beei Wu, Member, IEEE, and Tatsuo Itoh, Fellow, IEEE
}

\begin{abstract}
A hybrid finite-difference time-domain (FDTD) method is proposed for solving transient electromagnetic problems associated with structures of curved surfaces. The method employs the conventional FDTD method for most of the regular region but introduces the tetrahedral edge-based finite-element scheme to model the region near the curved surfaces. Without any interpolation for the fields on the curved surface, nor any additional stability constraint due to the finer division near the curved surfaces, the novel finite-element scheme is found to have second-order accuracy, unconditional stability, programming ease, and computational efficiency. The hybrid method is applied to solve the electromagnetic scattering of three-dimensional (3-D) arbitrarily shaped dielectric objects to demonstrate its superior performance.
\end{abstract}

Index Terms-Electromagnetic transient analysis, FDTD methods.

\section{INTRODUCTION}

$\mathbf{N}$ EEDLESS to say, finite-difference time-domain (FDTD) has become a prevalent method for solving various electromagnetic problems. In its original form, it works excellently for structures which can fit well into Cartesian coordinates [1]. The algorithm provides a lot of attractive advantages: direct and explicit time marching, high accuracy with discretization error of second order, obvious stability condition, easy programming, and minimum computational complexity.

One of the major disadvantages of FDTD is the inefficiency to model the structures with curved surfaces. To achieve accurate results using the staircase model for such structures usually requires a very fine grid and, consequently, a very small time step in time marching due to the stability constraint.

Exploiting conformity is one way to alleviate the staircasing approximation. Basically, there are three types of FDTD conformable surface models. The first type introduces a new curvilinear coordinate system to model globally the structure of interest [2], [3]. The scheme wastes a vast memory to store the coordinates and spends significantly larger computation time in updating the field, even in most of the regular region. To preserve the advantages of conventional FDTD as much as possible, the second type suggests the locally conformed scheme [4], [5]. Relying on the integral form of the Maxwell's equation, both types suffer from worse accuracy due to a larger

Manuscript received July 3, 1995; revised March 4, 1996. This work was supported by the National Science Council, R.O.C.

R.-B. Wu is with the Department of Electrical Engineering, National Taiwan University, Taipei, Taiwan, R.O.C.

T. Itoh is with the Electrical Engineering Department, University of California in Los Angeles, Los Angeles, CA 90024 USA.

Publisher Item Identifier S 0018-926X(97)05601-9. discretization error of first order. In addition, special attention should be paid so as not to make the algorithm unstable. The third type defines an additional local coordinate system near the curved surfaces and interpolates the field in the overlapping region [6]. Although with comparatively less stability concern, it involves interpolation back and forth, which deteriorates the accuracy and requires additional computation time.

The conformity approaches become cumbersome for structures difficult to be described by a certain well-known coordinate system globally or locally such as objects with corners or abrupt curvature changes. To better model the arbitrary boundary, the more flexible finite-element method (FEM) has been investigated recently [7], [8]. With a sophisticated management and vast memory storage for the bookkeeping system, FEM allows a very fine mesh locally to model complicated objects. The local fine mesh may cause a serious stability concern and consequently requires a small time step, which deteriorates the overall computational efficiency [7]. To free from the stability concern, it is suggested to employ an implicit scheme which requires the solution of a large system of simultaneous equations in each time step [8]. The matrix solution is quite time consuming even if the sparse matrix technique is employed. As a result, the computational complexity turns out to be the worst one among all the available approaches, i.e., $N^{1.5}$ versus $N^{1.33}$ where $N$ is the total number of unknowns.

In light of a larger computational load of FEM than that of FD, it will be advantageous to employs the conventional FDTD in most of the regular region and confine the FEM solution region near the curved surfaces only. This strategy can offer the best computational efficiency since it preserves all the FDTD advantages in most of the regular region while resorts to FEM just enough to model the curved surfaces. By employing the implicit scheme, the incorporated FEM can have unconditional stability such that the FEM mesh can be made arbitrarily fine locally without causing any stability problem. This idea has been successfully implemented for twodimensional (2-D) TM scattering problems [9]. It has been found to have good accuracy, stability, programming ease, and computational efficiency. This paper will implement this idea to three-dimensional (3-D) vector field problems.

\section{Hybrid TIME-MARChing SCHEME}

Consider a dielectric object of curved boundary shown in Fig. 1. The solution region is divided into the regular region and junction region. Although not shown in the figure, a uniform rectangular FDTD grid is employed in the regular 


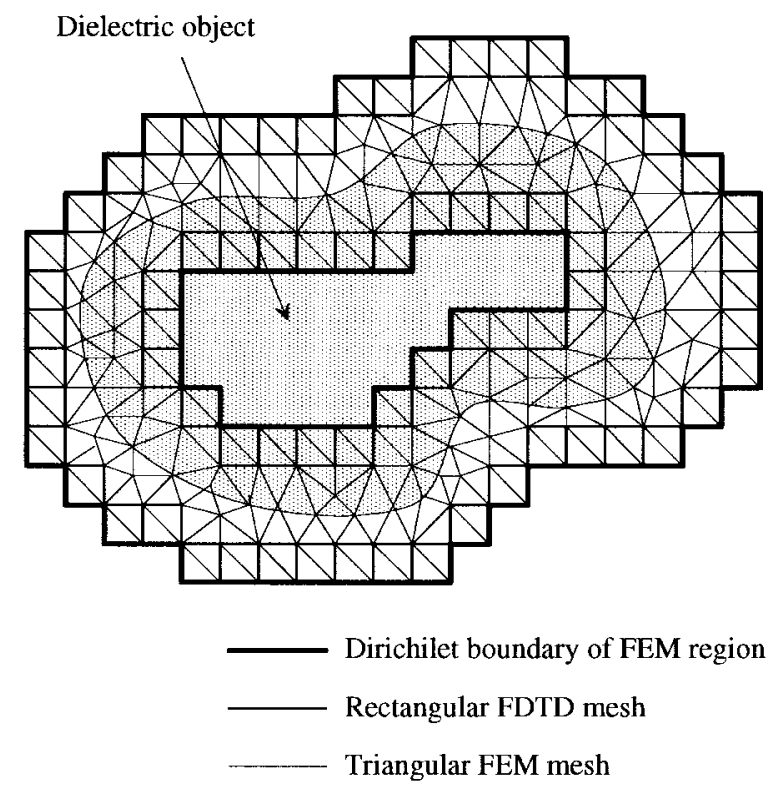

Fig. 1. FEM mesh in junction region and its interface with FDTD grid.

region where the structure fits well to the rectangular grid. In the present case, the regular region spans the exterior free space and the interior dielectric medium, both at a certain distance to the curved boundary. Depending on the division size in FDTD grid, the junction region usually spans four or five division units and encloses both sides of the curved boundary. Due to the flexibility offered by tetrahedral elements, it is not difficult to set up an FEM mesh in the junction region which makes the curved boundary conform to the FDTD grid. It is worth noting that both the exterior boundary of the junction region and the secondary boundary, which is one cell interior to the exterior boundary, exactly fit the FDTD grid.

Following FDTD conventions, the discretization is interleaved in both time and space. Let $\vec{H}_{\mathrm{FD}}^{n-\frac{1}{2}}$ and $\vec{E}_{\mathrm{FD}}^{n}$ denote the magnetic and electric fields, respectively, in the regular region. Here, the superscript stands for the time instant. Similarly, $\vec{E}_{\mathrm{FEM}}$ denotes the electric field in the junction region. Specifically, $\vec{E}_{\mathrm{FEM}, B}$ and $\vec{E}_{\mathrm{FEM}, I}$ are the electric fields on the exterior and secondary boundaries, respectively. It deserves mentioning that $\vec{E}_{\mathrm{FEM}, B}$ and $\vec{E}_{\mathrm{FEM}, I}$ coincide with the FDTD grid. Actually, the secondary boundary of the FEM region serves as the exterior boundary of the FDTD region, while the exterior boundary of the FEM region serves as the secondary boundary of the FDTD region.

Given all the electric fields $\vec{E}_{\mathrm{FD}}^{n}, \vec{E}_{\mathrm{FEM}, B}^{n}$, and $\vec{E}_{\mathrm{FEM}, I}^{n}$ at time instant $n$, one can update the magnetic field at time instant $n+\frac{1}{2}$ by applying the Faraday law [1]. The magnetic field in the regular region $\vec{H}_{\mathrm{FD}}^{n+1 / 2}$, including that on the center surface between exterior and secondary boundaries $\vec{H}_{\mathrm{FD}, I B}^{n+1 / 2}$, becomes available. From the obtained magnetic field, the electric field $\vec{E}_{\mathrm{FD}}^{n+1}$, the field in the regular region, and $\vec{E}_{\mathrm{FEM}, B}^{n+1}$, the field on the exterior boundary of the junction region, can be uniquely updated using the FDTD algorithm.

The electric field in the junction region at time instant $n+1$, including that on the secondary boundary, is obtained

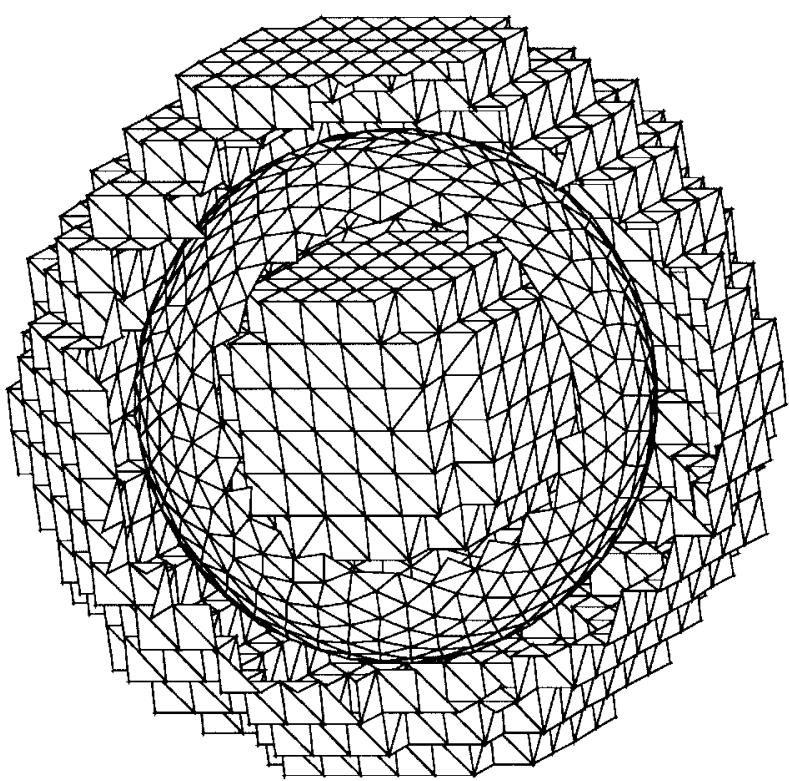

Fig. 2. Exterior boundary and object surface. The region in between is divided into tetrahedra.

by applying FEM. This is an initial value problem in time with previous $\vec{E}_{\text {FEM }}$ being the initial condition as well as a boundary value problem in space with $\vec{E}_{\mathrm{FEM}, B}^{n+1}$ being the Dirichlet boundary condition. Solution for this well-posed problem will be described in the next section. Once $\vec{E}_{\mathrm{FEM}}^{n+1}$ in the junction region becomes solved, together with the available $\vec{E}_{\mathrm{FD}}^{n+1}$ and $\vec{H}_{\mathrm{FD}}^{n+\frac{1}{2}}$ in the FDTD region, one can proceed with the time-marching scheme for the next time step and so on.

It is worth noting that the hybrid method follows the same FDTD algorithm except an embedded FEM processing for the region near the curved boundary, if any. This is quite advantageous in programming since almost all the endeavors already paid to develop the FDTD method need not be repeated again.

\section{SOLUTION OF MiXED-INITIAL AND BOUNDARY-VALUES PROBLEM}

\section{A. Mesh Generation}

It is well recognized that the tetrahedral elements are the most versatile to model 3-D structures of arbitrary shapes. The junction region to be solved by FEM has outer and inner hulls which are in a staircase shape due to the rectangular FDTD grid. A typical example is shown in Fig. 2 where the curved surface of the object lies in between. The junction region need be divided into a lot of well-sized tetrahedra under the constraint that none of them intersects the curved surface.

The Delaunay triangulation algorithm has been commonly employed to automatically construct the tetrahedral mesh in the 3-D space from the given nodal points [10]. The algorithm can be proved to be applicable theoretically if none of any five points lies on the same sphere. Direct application of the algorithm fails here since the junction region contains a lot of rectangular cells, for each of which the eight vertices are co-spherical. A modified algorithm has been suggested 
to circumvent this ambiguity by additionally enforcing the structure consistency in each triangulation step [11]. It is employed here and found to be efficient and robust.

\section{B. Variational Formulation}

Commonly expressed in differential or integral form, the Maxwell's equations can be cast as well into a variational form or the weak form [8], [11]. It states that the unknown field $\vec{E}(\vec{r}, t)$ in the junction region $\Omega$ is the very one which satisfies

$$
\frac{\partial^{2}}{\partial t^{2}} \int_{\Omega} \epsilon \vec{E}^{a} \cdot \vec{E} d \Omega=-\int_{\Omega} \frac{1}{\mu} \nabla \times \vec{E}^{a} \cdot \nabla \times \vec{E} d \Omega
$$

for arbitrary test field $\vec{E}^{a}(\vec{r}, t)$.

It is interesting to compare the present formula with that for the global FEM approach [8]. In global FEM approach, the formula includes an additional boundary term along the artificial lattice truncation $\Gamma_{\infty}$. As a result, one needs to devise the absorbing boundary condition solely for its use. In contrast, the present approach does not introduce any boundary term since the field along the boundary is already given by the FDTD time marching. At the lattice truncation, it applies directly the absorbing boundary condition whatever developed for conventional FDTD analysis. For simplicity, the Mur's first-order absorbing boundary condition [12] is employed in this paper.

\section{Edge-Based Vector Elements}

The variational formula should be discretized suitably to proceed with the numerical calculation. By FEM, suitable basis functions are chosen to represent the field inside each tetrahedral element. After taking volume integration inside each element and assembling the integrals from all the elements, (1) can be cast into a matrix form of

$$
[C] \frac{\partial^{2}}{\partial t^{2}} \bar{E}=-[D] \bar{E}
$$

where

$$
\begin{aligned}
& {[C]_{i j}=\int_{\Omega} \epsilon \vec{W}_{i} \cdot \vec{W}_{j} d \Omega} \\
& {[D]_{i j}=\int_{\Omega} \frac{1}{\mu} \nabla \times \vec{W}_{i} \cdot \nabla \times \vec{W}_{j} d \Omega}
\end{aligned}
$$

$\bar{E}$ denotes the coefficient vector for the unknown field and $\vec{W}_{i}(\vec{r})$ is the Whitney "one-from-edge" element [12].

Although written in the matrix form, the matrices $[C]$ and $[D]$ are symmetric and actually very sparse. The matrix dimension, i.e., the total number of FEM unknowns $N_{\mathrm{fem}}$, is about the same as the total number of elements $N_{\text {elm }}$. It is interesting to estimate the number of nonzero matrix terms. For each tetrahedral element, there are six edges and, thus, $6 \times 5 \div 2=15$ mutual terms. Among the mutual terms, three correspond to two nonconnecting edges while twelve correspond to two edges lying on a certain side of the tetrahedron. Each of the twelve terms shares the same matrix position with the mutual term between the two edges from another element. Hence, each element contributes $3+\frac{12}{2}=9$

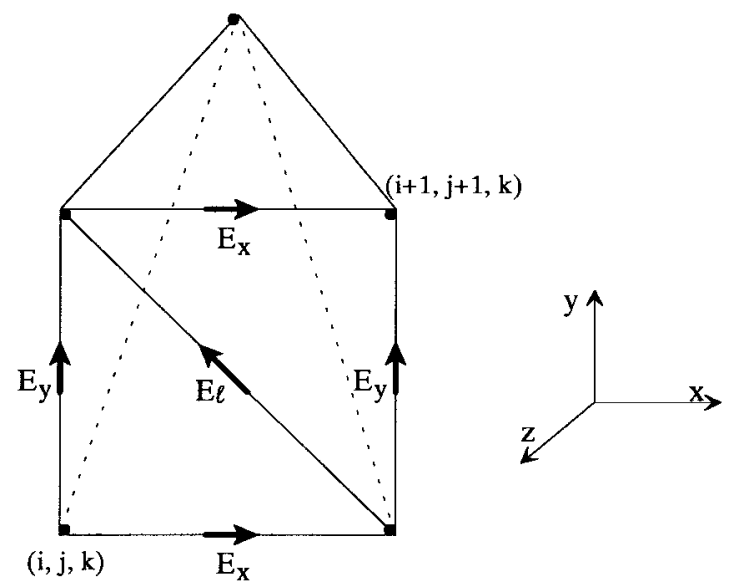

Fig. 3. Interface between rectangular FDTD mesh and tetrahedral FEM elements on exterior boundary surface.

mutual terms in the average. The number of nonzero terms need be stored for each matrix is at most $N_{\text {fem }}+9 N_{\text {elm }}$. To specify the sparse pattern, both matrices require an array for the indexes of those nonzero terms. As a result, the total storage for the two matrices is about $30 N_{\text {fem }}$.

Note that the test and unknown fields are expanded in terms of the same basis functions. This approach (also known as the Galerkin procedure) has a stationary nature and has been widely believed to be an accurate process with discretization error of second order [11].

The field variables in FDTD lie on a rectangular mesh while those in FEM lie on the tetrahedral edges. On the exterior boundary of the FEM region, the tetrahedral elements connect the rectangular mesh. Without loss of generality, consider a typical interface shown in Fig. 3. The tangential field along the edge on the boundary surface should be prescribed from the field variables given in the FDTD analysis. For the present case, the unit tangential vector along the edge is

$$
\hat{\ell}=\frac{1}{\sqrt{2}}(-\hat{x}+\hat{y}) \text {. }
$$

As a result, the field variable

$$
\begin{aligned}
E_{\ell}=\hat{\ell} \cdot \vec{E}\left(i+\frac{1}{2}, j+\frac{1}{2}, k\right) \\
=\frac{1}{2 \sqrt{2}}\left[-E_{x}\left(i+\frac{1}{2}, j, k\right)-E_{x}\left(i+\frac{1}{2}, j+1, k\right)\right. \\
\left.\quad+E_{y}\left(i, j+\frac{1}{2}, k\right)+E_{y}\left(i+1, j+\frac{1}{2}, k\right)\right] .
\end{aligned}
$$

Note that all the involved field variables are altogether in the same region. The interpolation (5) remains of the second-order accuracy - the same as the FDTD algorithm does.

\section{Newmark-Beta Scheme}

To solve the initial value problems in (2), the time derivative is usually approximated by the central difference, i.e.,

$$
\left.\frac{\partial^{2}}{\partial t^{2}} \bar{E}\right|_{t=n \Delta t} \approx \frac{1}{(\Delta t)^{2}}\left(\bar{E}^{n+1}-2 \bar{E}^{n}+\bar{E}^{n-1}\right) .
$$


After the discretization by (6), (2) reduces to a system of equations relating the unknown field $\vec{E}^{n+1}$ in the next time step to the given fields $\vec{E}^{n}$ and $\vec{E}^{n-1}$ in the present and past time steps. However, the matrix $[C]$ obtained by the Galerkin FEM is not diagonal. The updating scheme for the unknown field requires solution of the system of equations and is actually implicit. Besides, it will become unstable if the time step $\Delta t$ is larger than a certain value [8].

Investigation on the simple one-dimensional (1-D) scalar wave equation has indicated that the stability problem can be eliminated by introducing backward finite difference [14]. The Newmark-Beta scheme is a very popular one which formulates the field in right-hand side of (2) by [15]

$$
\left.\bar{E}\right|_{t=n \Delta t} \approx \frac{1}{4}\left(\bar{E}^{n+1}+2 \bar{E}^{n}+\bar{E}^{n-1}\right) .
$$

Substituting (6) and (7) into (2) yields the governing equations for the unknown field $\vec{E}^{n+1}$, i.e.,

$$
\begin{aligned}
& \left([C]+\frac{(\Delta t)^{2}}{4}[D]\right) \bar{E}^{n+1} \\
& \quad=2\left([C]-\frac{(\Delta t)^{2}}{4}[D]\right) \bar{E}^{n}-\left([C]+\frac{(\Delta t)^{2}}{4}[D]\right) \bar{E}^{n-1} .
\end{aligned}
$$

Given the boundary field $\vec{E}_{\mathrm{FEM}, B}^{n+1}$, all the field in the junction region $\vec{E}_{\mathrm{FEM}}^{n+1}$ can be solved from (8). Although with more complicated expression, the matrices in (8) have the same sparsity as the original ones in (2). To fully exploit the sparsity, the conjugate gradient method preconditioned with symmetric successive over relaxation operator (SSOR-CG) is employed to solve the unknown field [16].

\section{E. Unconditional Stability}

It is not difficult to show that (8) is unconditionally stable. The matrix $[C]$ is obviously positive definite. It is easy to show that $[D]$ is semi-positive since the volume integral in the right-hand side of (1) is nonnegative if the test field and the unknown field have the same basis functions.

The updating matrix in (8) can be defined as

$$
\begin{aligned}
{[A] } & \equiv\left([C]+\frac{(\Delta t)^{2}}{4}[D]\right)^{-1}\left([C]-\frac{(\Delta t)^{2}}{4}[D]\right) \\
& =\left([I]+\frac{(\Delta t)^{2}}{4}[C]^{-1}[D]\right)^{-1}\left([I]-\frac{(\Delta t)^{2}}{4}[C]^{-1}[D]\right)
\end{aligned}
$$

where $[I]$ denotes a unit matrix. It is clear that the two matrices $[A]$ and $[C]^{-1}[D]$ have the same eigenvectors. The eigenvalues $c_{j}$ s of $[C]^{-1}[D]$ must be nonnegative. Therefore, the eigenvalues $\lambda_{j}$ s of $[A]$ must have magnitude no larger than unity, since

$$
\left|\lambda_{j}\right|=\left|\frac{1-c_{j}}{1+c_{j}}\right| \leq 1 .
$$

Theoretically, the field $\bar{E}^{n}$ can be expanded in terms of the eigenvectors. The expansion coefficients of different eigenvectors are decoupled. Let $a_{n, j}$ denote the expansion coefficient corresponding to the $j$ th eigenvector. Substitution of the expansion coefficient to (8) yields the recurrence relation

$$
a_{n+1, j}-2 \lambda_{j} a_{n, j}+a_{n-1, j}=0 .
$$

Then the amplification factor can be shown to be

$$
\xi \equiv \frac{a_{n+1, j}}{a_{n, j}}=\lambda_{j} \pm i \sqrt{1-\lambda_{j}^{2}} .
$$

Obviously, it has a unit magnitude as that supposed for the lossless wave equation. This proves that the time marching by (8) is unconditionally stable. The stability condition of the hybrid method is determined solely from the FDTD algorithm in the regular region, which is already well known.

\section{COMPUTATIONAL COMPLEXITY}

It is interesting to investigate the computational complexity of the additional FEM processing as compared with the conventional FDTD method. This can be treated as the overhead of the FEM employed to improve the solution accuracy. This section will discuss the overhead in the computation time and required memory.

Consider a particular 3-D structure. Decreasing the FDTD division size $\Delta$ will increase the number of division $N_{\Delta}$ in each direction. The total number of variables $N$ is in the cubic order of $N_{\Delta}$ or written as $N=O\left(N_{\Delta}^{3}\right)$. The required memory for FDTD in the regular region will be $O(N)=O\left(N_{\Delta}^{3}\right)$. The mesh required to model the curved surface increases as well, while the mesh in the normal direction remains nearly the same. As a result, the total number of unknowns for FEM is $N_{\text {fem }}=O\left(N_{\Delta}^{2}\right)$. The required memory for the matrix storage is $O\left(N_{\text {fem }}\right)=O\left(N_{\Delta}^{2}\right)$. Hence, the FEM overhead in memory is $O\left(1 / N_{\Delta}\right)=O\left(N^{-\frac{1}{3}}\right)$.

As for the computation time, the total simulation time for the FDTD equals the number of time steps multiplied by the required computation time per time step. Assuming the physical time duration is fixed, the number of time steps increases proportionally to $N_{\Delta}$ as the division size $\Delta$ decreases. Hence, the total computation time for FDTD in the regular region is $O\left(N_{\Delta}^{4}\right)=O\left(N^{\frac{4}{3}}\right)$. The FEM preprocessing includes the housekeeping management for mesh, element integration, and matrix assembly. It need only be done once with the operation count proportional to $N_{\text {fem }}$. Hence, the required time is negligible as compared with the total simulation time.

During the time marching, the hybrid method needs to solve the system of equations in (8) by SSOR-CG once for each time step. Being an iterative method, the SSOR-CG in each iteration calls for a backward substitution for SSOR and a matrix multiplication [16], both of which require operation counts roughly the same as the number of nonzero elements in the matrix. Therefore, the computation time to solve (8) is proportional to $n_{\mathrm{CG}} N_{\mathrm{fem}}$ where $n_{\mathrm{CG}}$ is the number of iterations to converge.

For a bulk region divided into $N_{\text {fem }}$ unknowns and solved by diagonally preconditioned conjugate gradient method (PCCG), it was shown that $n_{\mathrm{CG}}$ is proportional to $\sqrt{N_{\text {fem }}}$ [8]. In the present case, the region of interest is a thin shell with thickness of four or five division cells, nearly independent of 


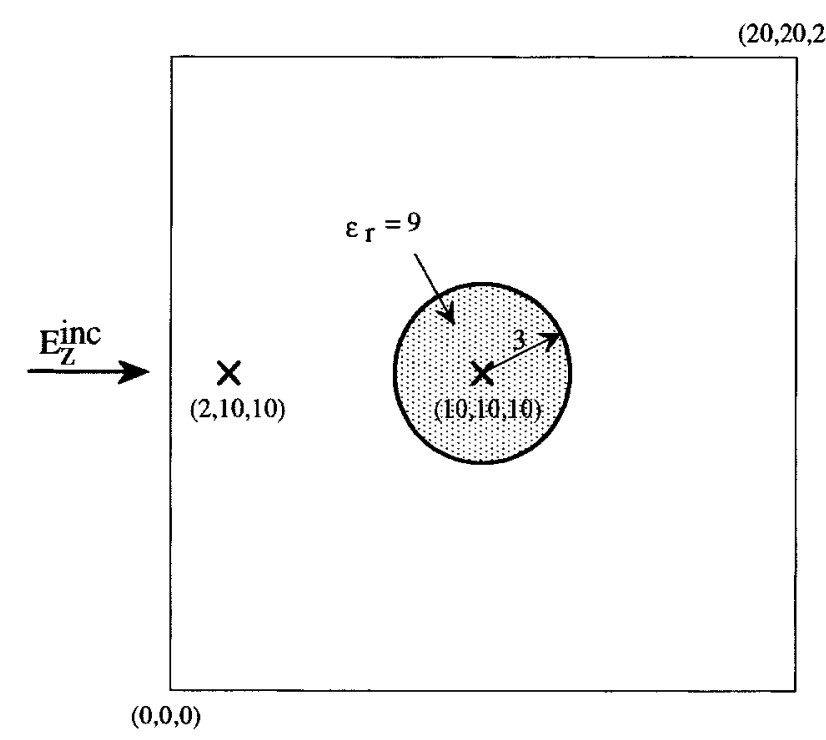

Fig. 4. A spherical dielectric scatterer illuminated by an incident $\hat{z}$-polarized Gaussian plane wave.

$N_{\text {fem }}$; besides, SSOR-CG converges faster than PC-CG [16]. Thus, it is expected that the iteration number $n_{\mathrm{CG}}$ should be smaller. Numerical experiments have been performed and, as to be described later, they show that $n_{\mathrm{CG}}$ varies more smoothly than $\sqrt{N_{\text {fem }}}$ does. As a result, the FEM overhead in computation time turns out to be smaller as $N$ increases.

\section{NUMERICAL RESULTS}

To investigate its numerical aspects, the hybrid time-domain method is applied to the electromagnetic scattering of 3-D arbitrarily shaped dielectric objects. In the numerical examples, the dielectric scatterers are illuminated by a $\hat{z}$-polarized plane wave of Gaussian pulse from $-\hat{x}$ direction, i.e.,

$$
\vec{E}^{\mathrm{inc}}(\vec{r}, t)=\hat{z} \exp \left\{-\frac{1}{2}\left(\frac{3(c t-x)}{c T}-5\right)^{2}\right\}
$$

where $c T$ denotes the $3 \sigma$ half width in space and is chosen $c T=10$ in the simulations. Note that $c$ is the velocity of light in vacuum such that $c T$ has the same unit as the space coordinates.

Fig. 4 shows the solution region for a spherical scatterer of dielectric constant $\epsilon_{r}=9$ and radius three unit. An automatic mesh generator is developed to construct the FEM mesh, which conforms the curved surface to the FDTD grid. For example, Fig. 2 shows the resultant mesh on the exterior boundary and the spherical surface for the case where the division size in FDTD grid is chosen as $\Delta=\frac{1}{2}$ unit.

Fig. 5 shows the results calculated by the hybrid method with the time-step ratio $\rho=\frac{c \Delta_{t}}{\Delta}=0.5$ and the FDTD division size $\Delta=1$ or $\frac{1}{2}$ as a parameter. Fig. 5(a) shows the total field at the sphere center $(10,10,10)$ and Fig. 5(b) shows the scattered field at the point $(2,10,10)$. Although the FEM division size may be smaller than $\Delta$ in the junction region, the present method will not encounter any stability problem only if $\rho$ is chosen smaller than $\frac{1}{\sqrt{3}}$. The solid curves shown in the figure denote the analytic results obtained by using the Mie

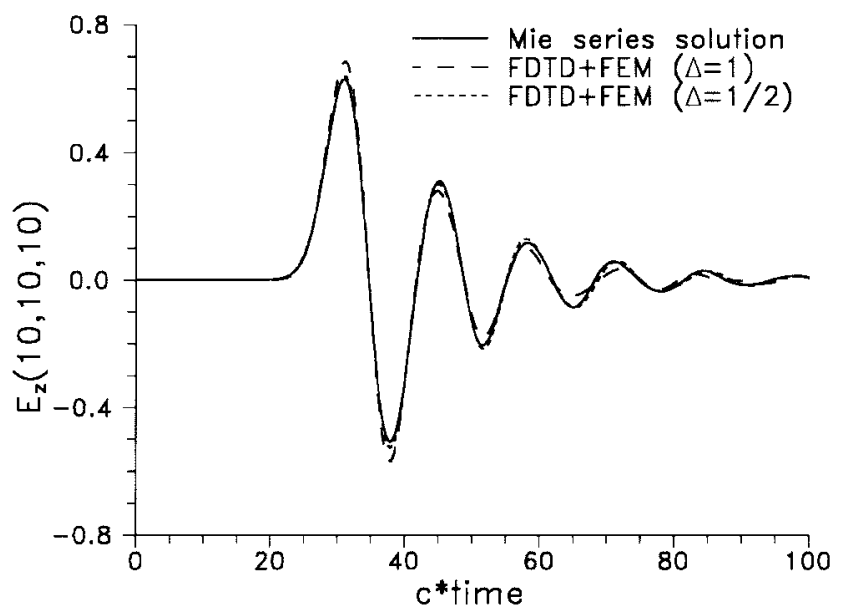

(a)

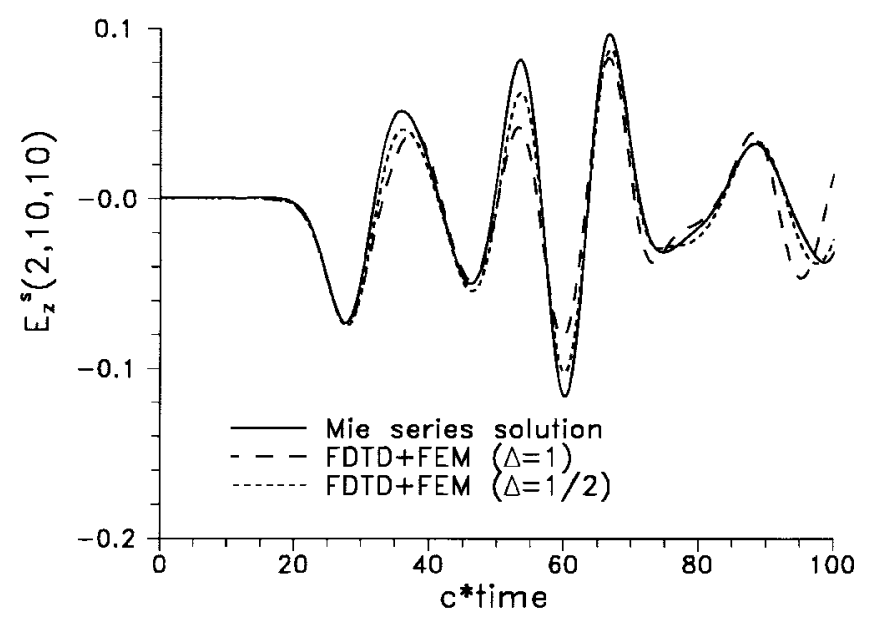

(b)

Fig. 5. Scattering due to a dielectric sphere shown in Fig. 4. Convergency of the calculated results by hybrid method as opposed to the analytic results obtained by Mie series solution. (a) Total field at the sphere center. (b) Scattered field at point $(2,10,10)$.

series solution [17]. It can be seen how well the numerical results converge to the analytic results as the division size decreases.

For the sake of comparison, Fig. 6 shows the results obtained by the conventional FDTD algorithm with a simple staircase approximation. The cells, which include the airdielectric interface, are assumed to have the average dielectric constant of $\frac{\epsilon_{r}+1}{2}$. As compared with the analytic results (solid curves), the results obtained by FDTD with staircase approximation still show the correct convergency tendency. However, comparison between Figs. 5 and 6 clearly demonstrates that its convergency is much worse than that of the present hybrid method. It seems that the hybrid method with a rough division of $\Delta=1$ can even yield better results than those achievable by the staircase FDTD analysis with a finer division of $\Delta=\frac{1}{2}$.

It is interesting to compare the overhead of the present hybrid method to the conventional FDTD algorithm. The incorporation of FEM requires additional computation time and memory in both the preprocessing and time-marching stages. In the case of $\Delta=1$, the regular FDTD of grid size $20 \times 20 \times 20$ requires memory storage of $0.29 \mathrm{Mb}$ and takes 


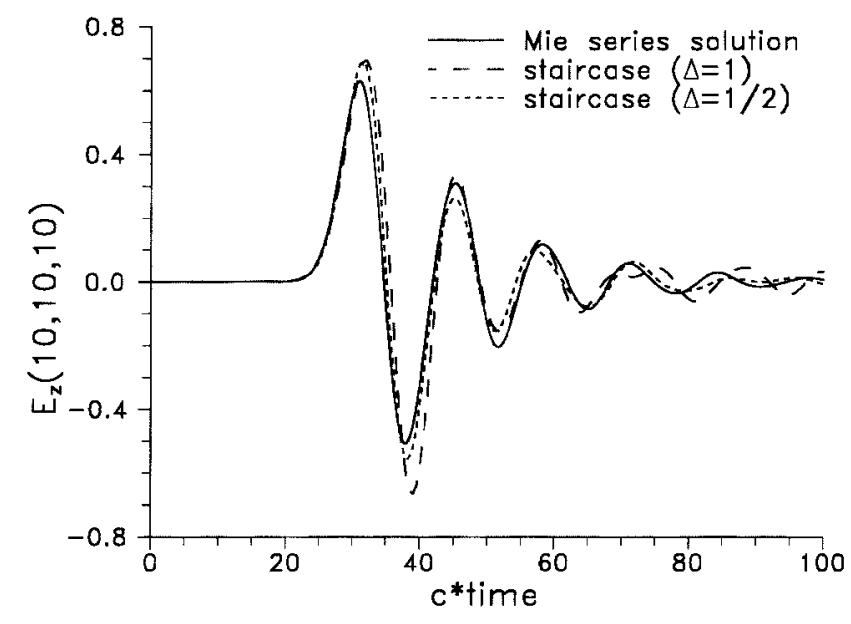

(a)

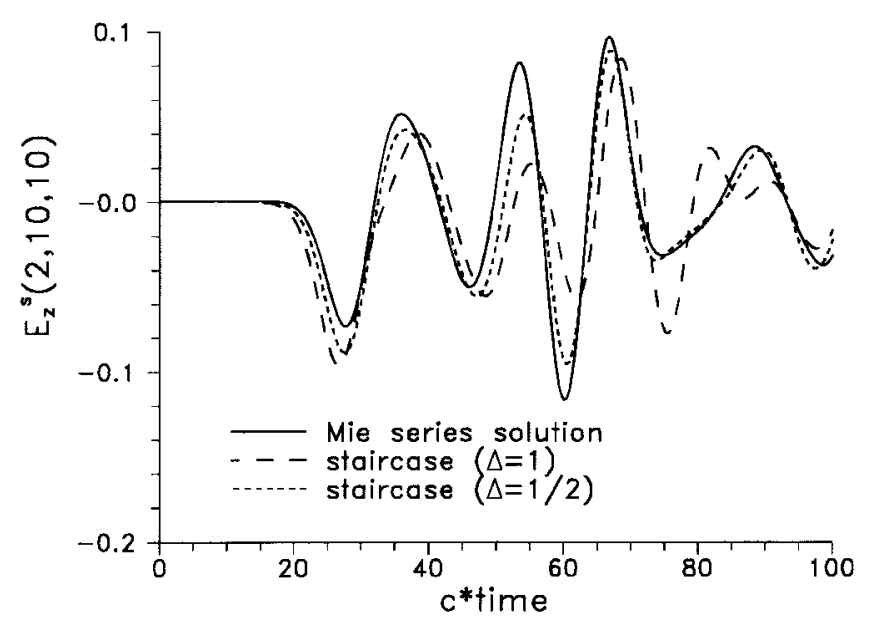

(b)

Fig. 6. Calculated results obtained by rectangular FDTD with staircase approximation to handle the scattering problem shown in Fig. 4. (a) Total field at the sphere center. (b) Scattered field at point $(2,10,10)$.

$0.31 \mathrm{~s}$ per time step as measured in a laptop IBM/PC486 with DX2-66 MHz clock rate. The hybrid method requires memory storage of $0.67 \mathrm{Mb}$ and takes $7.14 \mathrm{~s}$ for the FEM preprocessing. Most of the memory storage can be released for the timemarching stage. As a result, the FEM overhead in the time marching is $0.59 \mathrm{Mb}$ in memory and $1.11 \mathrm{~s}$ per time step, about 2.0X and 3.6X, respectively, as compared with those required in the FDTD analysis.

As the division size reduces by half, i.e., $\Delta=\frac{1}{2}$, the number of divisions along each direction is doubled. The total number of unknowns is multiplied by eight. The regular FDTD, now of size $40 \times 40 \times 40$ requires $1.92 \mathrm{Mb}$ and $2.12 \mathrm{~s}$ per time step, roughly eight times larger. In contrast, the FEM preprocessing now requires $2.46 \mathrm{Mb}$ and $21.59 \mathrm{~s}$, roughly four times larger. Also, the FEM overhead in the time marching is found to be $2.19 \mathrm{Mb}$ in memory and $5.51 \mathrm{~s}$ per time step, or $1.1 \mathrm{X}$ and $1.6 \mathrm{X}$, respectively. This demonstrates that the FEM overhead becomes smaller for complicated structures which involve a larger number of unknowns.

It is interesting to compare the iteration count $n_{\mathrm{CG}}$ between SSOR-CG and PC-CG. For the same convergency bound of

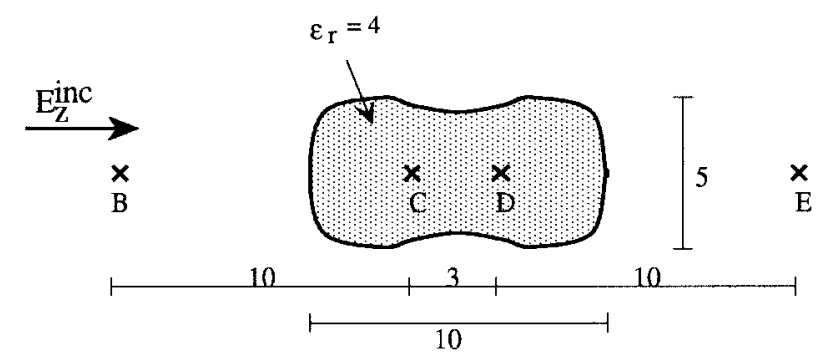

(a)

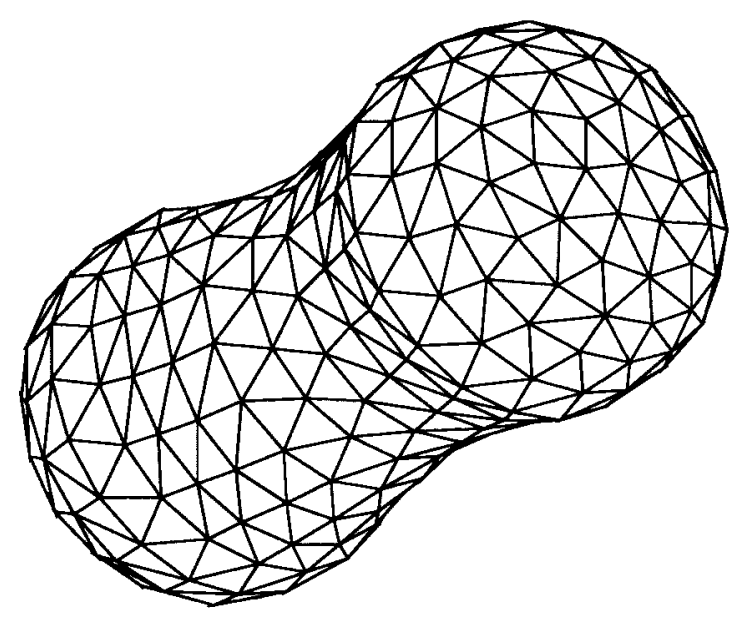

(b)

Fig. 7. Electromagnetic scattering of a peanut-like dielectric object. (a) Scatterer and observation points. (b) Mesh division on the curved surface.

$10^{-6}, n_{\mathrm{CG}}$ for SSOR-CG and PC-CG is found to be no larger than 7 and 16, respectively, in case of the coarse division. The number increases to 10 and 21 , respectively, in case of the fine division. Since SSOR-CG takes nearly twice the computing time than PC-CG does in each iteration, the overall computational time is nearly the same for these two iteration approaches.

The second example considers a peanut-like dielectric scatterer of dielectric constant $\epsilon_{r}=4$. Fig. 7(a) shows the solution region and (b) shows the mesh division on the scatterer surface while the division size in FDTD is $\Delta=1$. Fig. 8 shows the calculated scattered fields at points B, E and the total fields at points $\mathrm{C}, \mathrm{D}$ inside the scatterer.

Fig. 9 shows another example where the scatterer of $\epsilon_{r}=9$ has a cone. Fig. 10 plots the calculated scattered fields at points $\mathrm{B}, \mathrm{D}$ and the total fields at the point $\mathrm{C}$. Both the two polarizations of the scattered field at point $\mathrm{D}$ are illustrated. It is found that both the co- and cross-polarization of the cone-shaped objects are quite noticeable. Also, the late-time response at point $\mathrm{C}$ lasts for quite a long time. This depicts a strong multiple reflection inside the dielectric scatterer.

\section{CONCLUSION}

A hybrid time-domain method is proposed in this paper and is applied to deal with the electromagnetic scattering from 3-D arbitrarily shaped dielectric objects. This method hybridizes the efficient FDTD analysis for most of the regular region with the flexible FEM analysis for the junction region 


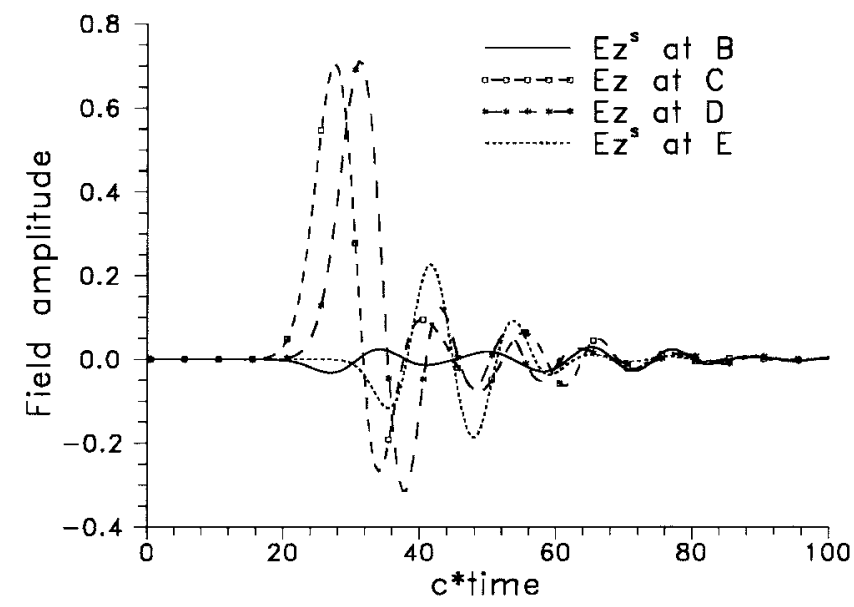

Fig. 8. Calculated scattered fields at points B, E and total fields at points C, D, shown in Fig. 7(a).

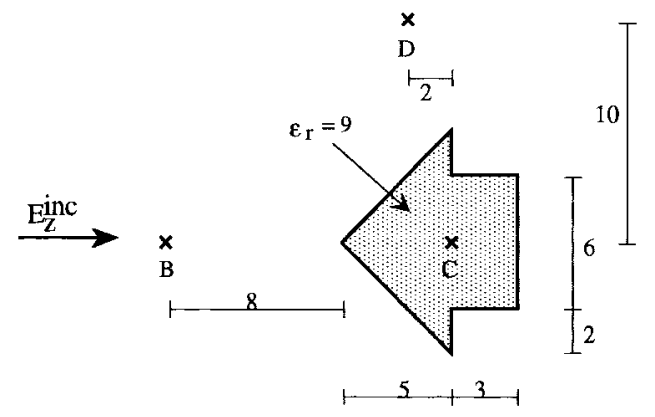

(a)

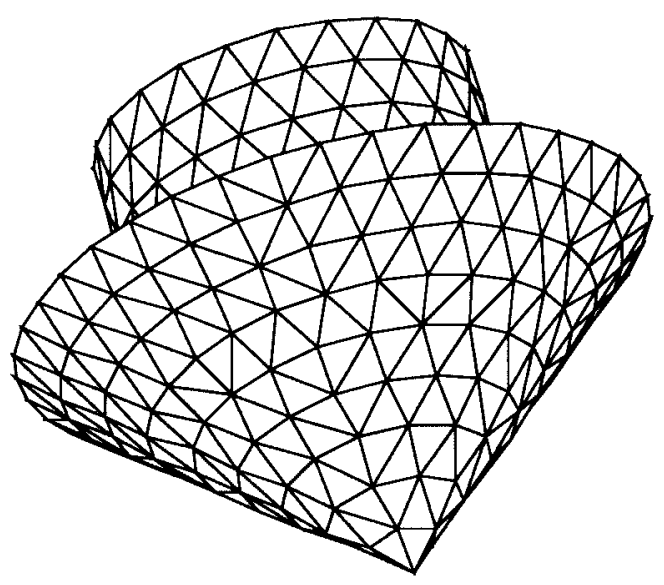

(b)

Fig. 9. Electromagnetic scattering of a cone-shaped dielectric object. (a) Scatterer and observation points. (b) Mesh division on the curved surface.

near the curved surface of the dielectric object. Using a lot of tetrahedral elements, which conform the arbitrary curved surface to the FDTD grid, the method does not resort to any interpolation for the fields on the curved surface. Also, it applies the Galerkin approach with edge elements to solve the FEM region. These, altogether, assure that the method keeps the same order of accuracy in curved structures as the conventional FDTD has in rectangular structures.

The time-marching formulation in the FEM region is based on the Newmark-Beta scheme, which is unconditionally sta-

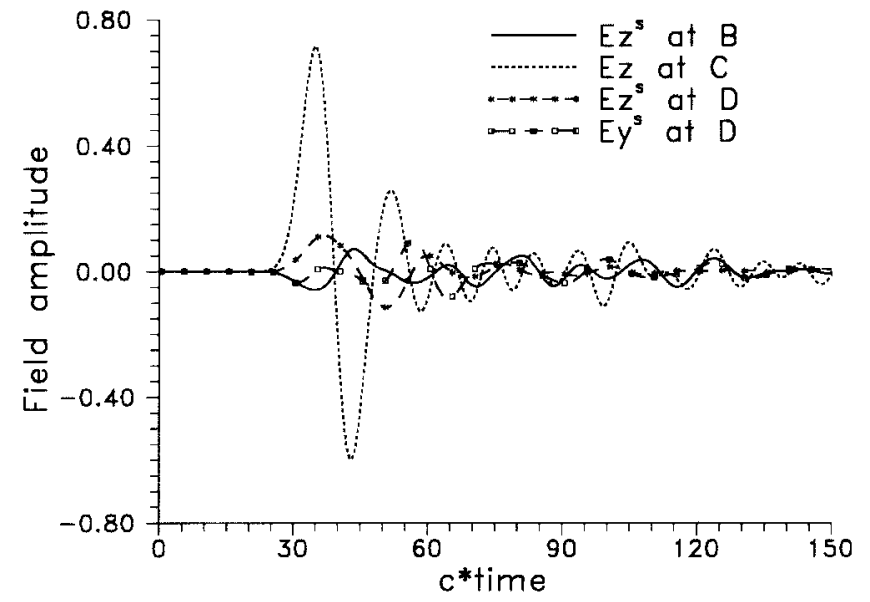

Fig. 10. Calculated scattered fields at points B, D and total field at point C, shown in Fig. 9(a).

ble. Consequently, there is no stability concern due to the perhaps extraordinary fine FEM mesh division required to model objects with corners of small curvature. In addition, the conjugate gradient method with symmetric successive overrelaxation preconditioning is employed to update the electric field in the FEM region. The involved computation time and memory storage are both comparable to those required for the FDTD analysis in the regular region. Hence, the present hybrid method is more computationally efficient than solely using the FDTD with the necessary highly refined grid to yield the same degree of accuracy.

Several examples have been presented to demonstrate the applications of the hybrid method for 3-D scattering problems. Numerical results validate that the method has the desired advantages of accuracy, flexibility, stability, and computational efficiency.

\section{REFERENCES}

[1] K. S. Yee, "Numerical solution of initial boundary value problems in isotropic media," IEEE Trans. Antennas Propagat., vol. AP-14, pp. 302-307, May 1966.

[2] R. Holland, "Finite-difference solutions of Maxwell's equations in generalized nonorthogonal coordinates," IEEE Trans. Nucl. Sci., vol. NS-30, pp. 4589-4591, 1983.

[3] J. F. Lee, R. Palandech, and R. Mittra, "Modeling three-dimensional discontinuities in waveguides using nonorthogonal FDTD algorithm," IEEE Trans. Microwave Theory Tech., vol. 40, pp. 346-352, Feb. 1992.

[4] T. G. Jurgens, A. Talflove, K. Umashanker, and T. G. Moore, "Finitedifference time-domain modeling of curved surfaces," IEEE Trans. Antennas Propagat., vol. 40, pp. 357-366, Apr. 1992.

[5] J. Fang and J. Ren, "A locally conformed finite-difference time-domain algorithm of modeling arbitrary shape planar metal strips," IEEE Trans. Microwave Theory Tech., vol. 41, pp. 830-838, May 1993.

[6] K. S. Yee, J. S. Chen, and A. H. Chang, "Conformal finite-difference time-domain (FDTD) with overlapping grid," IEEE Trans. Antennas Propagat., vol. 40, pp. 1068-1075, Sept. 1992.

[7] C. H. Thng and R. C. Booton, "Edge-element time-domain method for solving Maxwell's equations," in IEEE MTT-S Dig., May 1994, vol. 2, pp. 693-696.

[8] J. F. Lee and Z. Sacks, "Whitney elements time domain (WETD) methods," IEEE Trans. Magn., vol. 31, pp. 1325-1329, May 1995.

[9] R. B. Wu and T. Itoh, "Hybridizing FDTD analysis with unconditionally stable FEM for objects of curved boundary," in IEEE MTT-S Dig., May 1995, vol. 2, pp. 833-836.

[10] D. F. Watson, "Computing the $n$-dimensional Delaunay tessellation with applications to Voronoi polytopes," Comput., vol. 24, no. 2, pp. 167-172, 1981. 
[11] R. B. Wu, "A wideband waveguide transition design with dielectric transformer using edge-based tetrahedral finite element analysis," IEEE Trans. Microwave Theory Tech., vol. 44, pp. 1024-1031, July 1996.

[12] G. Mur, "Absorbing boundary conditions for the finite-difference approximation of the time-domain electromagnetic-field equation," IEEE Trans. Electromagn. Compat., vol. EMC-23, pp. 377-382, Nov. 1981.

[13] A. Bossavit, "Solving Maxwell's equations in a closed cavity and the question of spurious modes," IEEE Trans. Magn., vol. 25, pp. 702-705, Mar. 1990.

[14] L. Lapidus and G. F. Pinder, Numerical Solution of Partial Differential Equations in Science and Engineering. Princeton, NJ: Princeton Univ. Press, 1981, p. 493.

[15] N. M. Newmark, "A method of computation for structural dynamics," J. Eng. Mech. Div., ASCE, vol. 85, pp. 67-94, July 1959.

[16] H. R. Schwarz, Finite Element Methods. New York: Academic, 1988, sec. 4.7.

[17] R. F. Harrington, Time Harmonic Electromagnetic Fields. New York: McGraw-Hill, 1961, secs. 6-9.

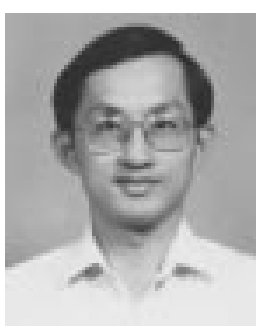

Ruey-Beei Wu (M'91) was born in Tainan, Taiwan, R.O.C., in 1957. He received the B.S.E.E. and Ph.D. degrees from National Taiwan University, Taipei, Taiwan, in 1979 and 1985, respectively.

In 1982, he joined the faculty of the Department of Electrical Engineering, National Taiwan University, Taipei, where he is now a Professor. He was a Visiting Scholar at IBM East Fishkill Facility, East Fishkill, NY, from 1986 to 1987 and in the Electrical Engineering Department, University of California, Los Angeles, from 1994 to 1995. His areas of interest include computational electromagnetics, dielectric waveguides, edge-slot antennas, wave-scattering of composite materials, transmission line and waveguide discontinuities, and interconnection modeling for computer packaging.

Tatsuo Itoh (S'69-M'69-SM'74-F'82), for photograph and biography, see this issue, p. 1301. 